\title{
New Cytotoxic Alkylated Anthraquinone Analogues from a Soil Actinomycete Streptomyces sp. WS-13394
}

\author{
Zhao-Yuan Wu, , Wei Fang, ${ }^{\#}$ Li-Qiao Shi, Zhong-Yi Wan, Yong Min, and Kai-Mei Wang* \\ Hubei Biopesticide Engineering Research Centre, Hubei Academy of Agricultural Sciences; \\ Wuhan 430064, P. R. China. \\ Received September 4, 2013; accepted September 30, 2013
}

Four new alkylated anthraquinone analogues (1-4) were isolated from a soil actimomycete Streptomyces sp. WS-13394. The structures of compounds 1-4 were elucidated to be 1,4,6-trihydroxy-8-alkylanthraquinones by means of spectroscopic methods, including UV, one dimensional (1D), 2D-NMR and MS spectrometry. All compounds showed activities against BGC-823 and MCF-7 with $\mathrm{IC}_{50}$ from 0.99 to $3.54 \mu \mathrm{g} /$ $\mathrm{mL}$, while 2 exhibited cytotoxicity against $\mathrm{HepG} 2, \mathrm{A875}, \mathrm{BGC}-823$ and $\mathrm{MCF}-7$ with $\mathrm{IC}_{50} 2.29,4.90,0.99$, and $1.66 \mu \mathrm{g} / \mathrm{mL}$, respectively.

Key words alkylated anthraquinone; Streptomyces sp. WS-13394; actinomycete; cytotoxic

Anthraquinones, having a wide distribution in nature, were well-known for their antimicrobial, antitumor, antiinflammatory and antiviral activities. ${ }^{1)}$ As reported in the literature, some of the alkylated 9,10-anthraquinone showed significant activity of antitumor, such as R1128 B, isolated from the cultured broth of Streptomyces sp. No. 1128, showed antitumor activites against MCF-7 (human breast adenocarcinoma cells) both xenografted to nude mice and implanted in subrenal capsule of mice, ${ }^{2}$ rhodoptilometrin and 3-propyl1,6,8-trihydroxy-9,10-anthraquinone from Echinoderm Colobometra perspinosa, showed non-selective activity towards MCF-7, SF-268 (central nervous system-glioblastoma cells), and H46 (lung large-cell carcinoma cells), ${ }^{3)}$ lupinacidina A-C from Micromonospora lupine, ${ }^{4,5)}$ were found to show significant inhibitory effects on the invasion of murine colon 26-L5 carcinoma cells without inhibiting cell growth, and galvaquinone B, isolated from a marine-derived Streptomyces spinoverrucosus, ${ }^{6}$ exhibiting epigenetic modulatory activity at $1.0 \mu \mathrm{M}$. In our search for secondary metabolites with antitumor activities from microorganism cultures, four new alkylated 9,10-anthraquinone analogues (1-4, Fig. 1) were isolated from a soil actinomycete Streptomyces sp. WS-13394. In this paper, we described the isolation, structure elucidation, and the cytotoxicity of compounds $\mathbf{1}-\mathbf{4}$.

\section{Results and Discussion}

Compound 1, obtained as a red pigment, had a molecular formula of $\mathrm{C}_{17} \mathrm{H}_{14} \mathrm{O}_{5}$, inferred by its high resolution-electronspray ionization-mass spectra (HR-ESI-MS) $(\mathrm{m} / \mathrm{z}$ 321.0737,

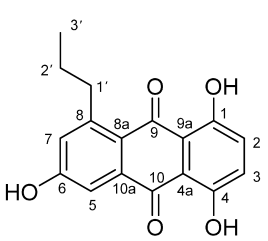

1

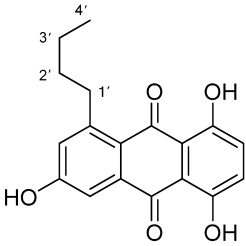

2

$\left.[\mathrm{M}+\mathrm{Na}]^{+}\right)$, requiring eleven degrees of unsaturation. The UV spectrum of 1 exhibited absorption bands at 466, 277, and $225 \mathrm{~nm}$, highly suggesting an anthraquinone chromophore. ${ }^{6}$ The ${ }^{1} \mathrm{H}-\mathrm{NMR}$ spectrum of $\mathbf{1}$ (Table 1) showed two orthocoupling aromatic protons at $\delta_{\mathrm{H}} 7.24(1 \mathrm{H}, \mathrm{d}, J=9.3 \mathrm{~Hz}, \mathrm{H}-2)$ and $7.20(1 \mathrm{H}, \mathrm{d}, J=9.3 \mathrm{~Hz}, \mathrm{H}-3)$, and two meta-coupling ones at $\delta_{\mathrm{H}} 7.57(1 \mathrm{H}, \mathrm{d}, J=2.5 \mathrm{~Hz}, \mathrm{H}-5)$ and $6.98(1 \mathrm{H}, \mathrm{d}, J=2.5 \mathrm{~Hz}$, $\mathrm{H}-7)$. In addition, a propyl moiety contributed by two methylene signals at $\delta_{\mathrm{H}} 3.15\left(2 \mathrm{H}, \mathrm{t}, J=7.7 \mathrm{~Hz}, \mathrm{H}-1^{\prime}\right)$ and $1.67(2 \mathrm{H}, \mathrm{m}$, $\left.\mathrm{H}-2^{\prime}\right)$, and a methyl signal at $\delta_{\mathrm{H}} 1.01\left(3 \mathrm{H}, \mathrm{t}, J=7.3 \mathrm{~Hz}, \mathrm{H}-3^{\prime}\right)$ were also observed in the ${ }^{1} \mathrm{H}-\mathrm{NMR}$ spectrum. In the ${ }^{13} \mathrm{C}-\mathrm{NMR}$ spectrum of 1 (Table 2), $14 s p^{2}$ carbon signals, including four quaternary $s p^{2}$ carbon signals in the low-field region at $\delta_{\mathrm{C}}$ $152.0,158.1,158.2$ and 164.2, and two carbonyl carbon signals at $\delta_{\mathrm{C}} 188.2$ and 189.2, suggesting the presence of a tetrasubstituted anthraquinone core. As required by the molecular formula, three hydroxyl groups and a propyl group account for the substituents on the anthraquinone ring.

The substituents and their location on the anthraquinone ring were further established by analysis of the correlation spectroscopy (COSY) and heteronuclear multiple bond correlation (HMBC) spectra of $\mathbf{1}$ (Fig. 2). COSY correlations between three aliphatic protons $\left(\mathrm{H}-1^{\prime} / \mathrm{H}-2^{\prime}\right.$ and $\left.\mathrm{H}-2^{\prime} / \mathrm{H}-3^{\prime}\right)$, combined with HMBC correlations from $\mathrm{H}-1^{\prime}$ to $\mathrm{C}-2^{\prime}$ (25.1), C-3' (14.7), C-8 (152.0) and C-8a (123.9), and $\mathrm{H}-2^{\prime}$ to $\mathrm{C}-1^{\prime}$ (39.3), C-3' and C-8 established the location of a propyl group at $\mathrm{C}-8$, which in turn indicated the hydroxy groups were located at C-1, C-4 and C-6, respectively. This deduction was corroborated by the HMBCs of H-2 and H-3 to C-1 (158.2)<smiles>CC(F)(F)CCc1cc(O)cc2c1C(=O)c1c(O)ccc(O)c1C2=O</smiles>

3<smiles>CCCCCc1cc(O)cc2c1C(=O)c1c(O)ccc(O)c1C2=O</smiles>

4

Fig. 1. Structures of Compounds $\mathbf{1}-\mathbf{4}$

The authors declare no conflict of interest.

"These authors contributed equally to this work. 
Table 1. ${ }^{1} \mathrm{H}-\mathrm{NMR}(500 \mathrm{MHz})$ Data of Compounds $\mathbf{1}-\mathbf{4}$ in $\mathrm{CD}_{3} \mathrm{OD}\left(\delta_{\mathrm{H}}, J\right.$ in $\left.\mathrm{Hz}\right)$

\begin{tabular}{cllll}
\hline \hline No. & \multicolumn{1}{c}{$\mathbf{1}$} & \multicolumn{2}{c}{$\mathbf{3}$} & $\mathbf{4}$ \\
\hline $2^{a)}$ & $7.24(1 \mathrm{H}, \mathrm{d}, J=9.3)$ & $7.17(1 \mathrm{H}, \mathrm{d}, J=9.2)$ & $7.16(1 \mathrm{H}, \mathrm{d}, J=9.3)$ & $7.22(1 \mathrm{H}, \mathrm{d}, J=9.3)$ \\
$3^{a)}$ & $7.20(1 \mathrm{H}, \mathrm{d}, J=9.3)$ & $7.13(1 \mathrm{H}, \mathrm{d}, J=9.2)$ & $7.11(1 \mathrm{H}, \mathrm{d}, J=9.3)$ & $7.18(1 \mathrm{H}, \mathrm{d}, J=9.3)$ \\
5 & $7.57(1 \mathrm{H}, \mathrm{d}, J=2.5)$ & $7.45(1 \mathrm{H}, \mathrm{d}, J=2.4)$ & $7.42(1 \mathrm{H}, \mathrm{d}, J=2.5)$ & $7.52(1 \mathrm{H}, \mathrm{d}, J=2.5)$ \\
7 & $6.98(1 \mathrm{H}, \mathrm{d}, J=2.5)$ & $6.89(1 \mathrm{H}, \mathrm{d}, J=2.4)$ & $6.86(1 \mathrm{H}, \mathrm{d}, J=2.5)$ & $6.94(1 \mathrm{H}, \mathrm{d}, J=2.5)$ \\
$1^{\prime}$ & $3.15(2 \mathrm{H}, \mathrm{t}, J=7.7)$ & $3.08(2 \mathrm{H}, \mathrm{t}, J=7.6)$ & $3.05(2 \mathrm{H}, \mathrm{m})$ & $3.12(2 \mathrm{H}, \mathrm{t}, J=7.7)$ \\
$2^{\prime}$ & $1.67(2 \mathrm{H}, \mathrm{m})$ & $1.57(2 \mathrm{H}, \mathrm{m})$ & $1.45(2 \mathrm{H}, \mathrm{m})$ & $1.64(2 \mathrm{H}, \mathrm{m})$ \\
$3^{\prime}$ & $1.01(3 \mathrm{H}, \mathrm{t}, J=7.3)$ & $1.47(2 \mathrm{H}, \mathrm{m})$ & $1.71(1 \mathrm{H}, \mathrm{m})$ & $1.46(2 \mathrm{H}, \mathrm{m})$ \\
$4^{\prime}$ & & $1.01(3 \mathrm{H}, \mathrm{t}, J=7.1)$ & $1.02(3 \mathrm{H}, \mathrm{d}, J=6.7)$ & $1.43(2 \mathrm{H}, \mathrm{m})$ \\
$5^{\prime}$ & & & $1.02(3 \mathrm{H}, \mathrm{d}, J=6.7)$ & $0.97(3 \mathrm{H}, \mathrm{t}, J=7.0)$ \\
\hline
\end{tabular}

a) Signals of H-2 and H-3 may be reversed.

Table 2. $\quad{ }^{13} \mathrm{C}-\mathrm{NMR}(125 \mathrm{MHz})$ Data of Compounds $\mathbf{1}-\mathbf{4}$ in $\mathrm{CD}_{3} \mathrm{OD}$

\begin{tabular}{|c|c|c|c|c|}
\hline No. & 1 & 2 & 3 & 4 \\
\hline $1^{a)}$ & $158.2 \mathrm{~s}$ & $158.0 \mathrm{~s}$ & $158.0 \mathrm{~s}$ & $158.2 \mathrm{~s}$ \\
\hline $2^{b)}$ & $129.0 \mathrm{~d}$ & $130.4 \mathrm{~d}$ & $130.1 \mathrm{~d}$ & $130.5 \mathrm{~d}$ \\
\hline $3^{b)}$ & $128.4 \mathrm{~d}$ & $128.3 \mathrm{~d}$ & $127.9 \mathrm{~d}$ & $128.3 \mathrm{~d}$ \\
\hline $4^{a)}$ & $158.2 \mathrm{~s}$ & $158.1 \mathrm{~s}$ & $158.0 \mathrm{~s}$ & $158.1 \mathrm{~s}$ \\
\hline $4 a^{c)}$ & $114.0 \mathrm{~s}$ & $113.9 \mathrm{~s}$ & $113.6 \mathrm{~s}$ & $114.0 \mathrm{~s}$ \\
\hline 5 & $113.3 \mathrm{~d}$ & $113.1 \mathrm{~d}$ & $112.7 \mathrm{~d}$ & $113.2 \mathrm{~d}$ \\
\hline 6 & $164.2 \mathrm{~s}$ & $163.7 \mathrm{~s}$ & $163.6 \mathrm{~s}$ & $163.8 \mathrm{~s}$ \\
\hline 7 & $125.8 \mathrm{~d}$ & $125.4 \mathrm{~d}$ & $125.9 \mathrm{~d}$ & $125.5 \mathrm{~d}$ \\
\hline 8 & $152.0 \mathrm{~s}$ & $152.2 \mathrm{~s}$ & $152.2 \mathrm{~s}$ & $152.3 \mathrm{~s}$ \\
\hline $8 a$ & $123.9 \mathrm{~s}$ & $123.9 \mathrm{~s}$ & $124.3 \mathrm{~s}$ & $124.0 \mathrm{~s}$ \\
\hline 9 & $189.2 \mathrm{~s}$ & $189.3 \mathrm{~s}$ & $189.0 \mathrm{~s}$ & $189.4 \mathrm{~s}$ \\
\hline $9 \mathrm{a}^{c)}$ & $114.8 \mathrm{~s}$ & $114.7 \mathrm{~s}$ & $114.3 \mathrm{~s}$ & $114.8 \mathrm{~s}$ \\
\hline 10 & $188.2 \mathrm{~s}$ & $188.4 \mathrm{~s}$ & $187.8 \mathrm{~s}$ & $188.5 \mathrm{~s}$ \\
\hline $10 \mathrm{a}$ & $137.4 \mathrm{~s}$ & $138.7 \mathrm{~s}$ & $138.4 \mathrm{~s}$ & $138.8 \mathrm{~s}$ \\
\hline $1^{\prime}$ & $39.3 \mathrm{t}$ & $36.9 \mathrm{t}$ & $34.9 \mathrm{t}$ & $37.2 \mathrm{t}$ \\
\hline $2^{\prime}$ & $25.1 \mathrm{t}$ & $34.1 \mathrm{t}$ & $40.9 \mathrm{t}$ & $31.6 \mathrm{t}$ \\
\hline $3^{\prime}$ & $14.7 \mathrm{q}$ & $24.1 \mathrm{t}$ & $29.6 \mathrm{~d}$ & $33.3 \mathrm{t}$ \\
\hline $4^{\prime}$ & & $14.3 \mathrm{q}$ & $22.6 \mathrm{q}$ & $24.0 \mathrm{t}$ \\
\hline $5^{\prime}$ & & & $22.6 \mathrm{q}$ & $14.5 \mathrm{q}$ \\
\hline
\end{tabular}

$a-c$ ) Signals of C-1 and C-4, C-2 and C-3, C-4a and C-9a may be reversed.

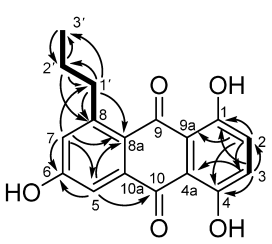

1

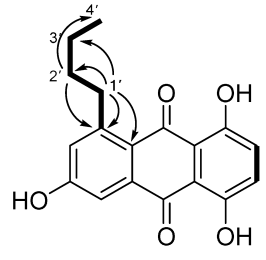

2

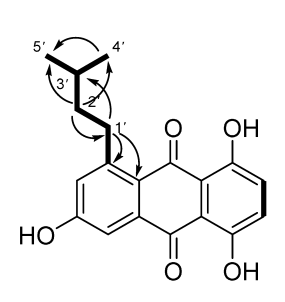

3

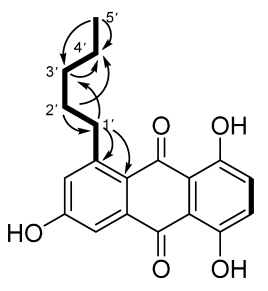

COSY

$\rightarrow \mathrm{HMBC}$

Fig. 2. COSY and Key HMBC Correlations for 1-4

and $\mathrm{C}-4$ (158.2), H-5 and H-7 to C-6 (164.2), and COSY correlations between $\mathrm{H}-2$ and $\mathrm{H}-3$. As a result, 1 was established to be 1,4,6-trihydroxy-8-propyl-9,10-anthraquinone.

Compound 2, isolated as red powder, had a molecular formula of $\mathrm{C}_{18} \mathrm{H}_{16} \mathrm{O}_{5}$, as established by HR-ESI-MS $(\mathrm{m} / \mathrm{z}$ 335.0894, $\left.[\mathrm{M}+\mathrm{Na}]^{+}\right)$, one more methylene $\left(\mathrm{CH}_{2}\right)$ than 1 . The ${ }^{1} \mathrm{H}$ - and ${ }^{13} \mathrm{C}$-NMR data were almost identical with those of $\mathbf{1}$ (Tables 1,2), except for the resonances for the alkyl substituent at C-8. NMR data (Tables 1,2$)$ of three methylenes $\left[\delta_{\mathrm{H}}\right.$ $3.08(2 \mathrm{H}, \mathrm{t}, J=7.6 \mathrm{~Hz}, \mathrm{H}-1), 1.57(2 \mathrm{H}, \mathrm{m}, \mathrm{H}-2)$ and $1.47(2 \mathrm{H}, \mathrm{m}$,
$\left.\mathrm{H}-3) ; \delta_{\mathrm{C}} 36.9,34.1,24.1\right]$ and a methyl $\left[\delta_{\mathrm{H}} 1.01(3 \mathrm{H}, \mathrm{t}, J=7.1)\right.$; $\left.\delta_{\mathrm{C}} 14.3 \mathrm{q}\right]$, together with COSY correlations (Fig. 2) between the aliphatic protons $\left(\mathrm{H}-1^{\prime} / \mathrm{H}-2^{\prime}, \mathrm{H}-2^{\prime} / \mathrm{H}-3^{\prime}\right.$, and $\left.\mathrm{H}-3^{\prime} / \mathrm{H}-4^{\prime}\right)$ showed the existence of a butyl group. HMBC correlations (Fig. 2) from $\mathrm{H}-\mathrm{l}^{\prime}$ to $\mathrm{C}-8$ (152.2) and C-8a (123.9) indicated that the butyl moiety was located at C- 8 . Therefore, the structure of 2 was established as 1,4,6-trihydroxy-8-propyl-9,10anthraquinone.

Compounds 3 and 4, both obtained as red powder, gave the same $[\mathrm{M}+\mathrm{Na}]^{+}$ion at $\mathrm{m} / \mathrm{z} 349.1051$ in HR-ESI-MS spectrum, 
Table 3. Cytotoxic Activity of the Compounds against Human Tumor Cells

\begin{tabular}{lrrrr}
\hline \hline \multirow{2}{*}{ Compd. No. } & \multicolumn{4}{c}{ In vitro cytotoxicity $\mathrm{IC}_{50}{ }^{a)}(\mu \mathrm{g} / \mathrm{mL})$} \\
\cline { 2 - 5 } & \multicolumn{1}{c}{$\mathrm{HepG2}^{b)}$} & $\mathrm{A} 875^{b)}$ & ${\mathrm{BGC}-823^{b)}}$ & \multicolumn{1}{c}{$\mathrm{MCF}^{b} 7^{b)}$} \\
\hline $\mathbf{1}$ & $9.48 \pm 1.34$ & $8.61 \pm 1.96$ & $3.54 \pm 0.25$ & $3.16 \pm 0.59$ \\
$\mathbf{2}$ & $2.29 \pm 0.21$ & $4.90 \pm 1.26$ & $0.99 \pm 0.04$ & $1.66 \pm 0.41$ \\
$\mathbf{3}$ & $23.61 \pm 4.73$ & $10.23 \pm 1.47$ & $1.76 \pm 0.18$ & $1.54 \pm 0.58$ \\
$\mathbf{4}$ & $8.72 \pm 1.56$ & $11.04 \pm 0.91$ & $3.08 \pm 0.83$ & $2.89 \pm 0.62$ \\
$\mathbf{5}$-Fluorouracil & $10.95 \pm 0.68$ & $13.26 \pm 1.12$ & $7.18 \pm 1.00$ & $13.94 \pm 2.62$ \\
\hline
\end{tabular}

a) $\mathrm{IC}_{50}$, compound concentration required to inhibit tumor cell proliferation by 50\%. b) Abbreviations: HepG2, human hepatocellular liver carcinoma cell line; A875, human malignant melanoma cell line; BGC-823, human gastric cancer cell line; MCF-7, human breast adenocarcinoma cell line. c) Used as a positive control.

which was consistent with the molecular formula of $\mathrm{C}_{19} \mathrm{H}_{18} \mathrm{O}_{5}$. Comparison of NMR spectra (Tables 1,2) data of compounds $\mathbf{3}$ and $\mathbf{4}$ with those of $\mathbf{1}$ and $\mathbf{2}$ indicated differences in the alkyl substituent at C-8. Five aliphatic carbon including two methylenes, one methenyl, and two methyls, together with the COSY correlations (Fig. 2) (H-1'/H-2', H-2'/H-3', H-3'/H-4' and $\left.\mathrm{H}-3^{\prime} / \mathrm{H}-5^{\prime}\right)$, suggested the existence of an isopentyl group in compound 3. In contrast, resonances attributed to aliphatic carbons of four methylenes and one methyl, and COSY correlations (Fig. 2) of $\mathrm{H}-\mathrm{1}^{\prime} / \mathrm{H}-2^{\prime} / \mathrm{H}-3^{\prime} / \mathrm{H}-4^{\prime} / \mathrm{H}-5^{\prime}$ showed the presence of a pentyl moiety in compound 4 . The full structures of $\mathbf{3}$ and $\mathbf{4}$ were further confirmed by heteronuclear single quantum coherence (HSQC) and HMBC experiments. Finally, structures of $\mathbf{3}$ and $\mathbf{4}$ were formulated as 1,4,6-trihydroxy8-isopentyl-9,10-anthraquinone and 1,4,6-trihydroxy-8-pentyl9,10-anthraquinone respectively.

Compounds 1-4 were examined for their cytotoxic activities against four human tumor cells lines. All compounds showed activities against BGC-823 and MCF-7 with $\mathrm{IC}_{50}$ from 0.99 to $3.54 \mu \mathrm{g} \cdot \mathrm{mL}^{-1}$, while 2 exhibited cytotoxicity against HepG2, A875, BGC-823 and MCF-7 with $\mathrm{IC}_{50}$ 2.29, 4.90, 0.99, and $1.66 \mu \mathrm{g} \cdot \mathrm{mL}^{-1}$ respectively (Table 3 ).

\section{Experimental}

General Experimental Procedures NMR spectra, including HSQC, HMBC and COSY, were recorded on a Bruker AVANCE-500 instrument with tetramethylsilane (TMS) as an internal standard (Bruker BioSpin group, German). ESIMS and HR-ESI-MS data were obtained on a Waters LC-MS (Waters Corporation, U.S.A.) and Thermo Q-T of Micromass (Thermo Electron Corporation, U.S.A.) spectrometers, respectively. Preparative HPLC was carried on a Waters 2767 Autopurification System (Waters Corporation, U.S.A.) coupled with a diode array detector (DAD) detector, using Sunfire Prep $\mathrm{C}_{18}$ OBD $(5 \mu \mathrm{m}, 19 \times 250 \mathrm{~mm} / 10 \times 250 \mathrm{~mm}$, Ireland $)$ column.

Microorganism The soil actinomycete WS-13394 was isolated from a soil sample collected from Shimen County, Hunan Province, China, in January 2007. Analysis of $16 \mathrm{~S}$ ribosomal RNA (rRNA) sequence of WS-13394 revealed 99\% identity to Streptomyces sp. VTT E-062996. A voucher strain was preserved at Hubei Biopesticide Engineering Research Center, Hubei Academy of Agricultural Sciences, in Wuhan, China.

Cultivation and Extraction Seed fermentations of strain WS-13394 were carried out in ISP-2 medium (glucose $4.0 \mathrm{~g} / \mathrm{L}$, malt extract $10.0 \mathrm{~g} / \mathrm{L}$, yeast extract $4.0 \mathrm{~g} / \mathrm{L}$, agar $20.0 \mathrm{~g} / \mathrm{L}$, ad- just $\mathrm{pH}$ 7.2) and shaken at $28^{\circ} \mathrm{C}$ at $120 \mathrm{rpm}$. After $96 \mathrm{~h}$ of cultivation, seed culture (10\%) was transferred to the Erlenmeyer flask $(500 \mathrm{~mL}$ containing $200 \mathrm{~mL}$ medium) with medium ISP-2 under sterile conditions, and incubated at $28^{\circ} \mathrm{C}$ for $120 \mathrm{~h}$ on a rotary shaker $(120 \mathrm{rpm})$. The culture $(20 \mathrm{~L})$ was extracted with $20 \mathrm{~L}$ ethyl acetate stirring $30 \mathrm{~min}$ for three times. The ethyl acetate was filtrated and then concentrated in vacuo to give a crude extract $(23.0 \mathrm{~g})$.

Isolation The ethyl acetate extract was dissolved in methanol and profiled with reversed-phased HPLC (Sunfire ${ }^{\circledR}$, Prep $\mathrm{C}_{18}$ OBD, $19 \times 250 \mathrm{~mm}, 5 \mu \mathrm{m}, 27 \mathrm{~mL} / \mathrm{min}$ ) using a gradient solvent system from $5-100 \% \mathrm{CH}_{3} \mathrm{CN}$ over $30 \mathrm{~min}$ to afford 11 fractions. The active fractions (Frs. 9-11) were further purified by semipreparative HPLC (Sunfire ${ }^{\circledR}$ Prep $\mathrm{C}_{18}$ OBD, $10 \times 250 \mathrm{~mm}, 5 \mu \mathrm{m}, 7.5 \mathrm{~mL} / \mathrm{min}$ ) using a gradient solvent system from 30 to $90 \% \mathrm{CH}_{3} \mathrm{CN}$ over $30 \mathrm{~min}$, to get compounds $1(3.44 \mathrm{mg})$ and $2(7.95 \mathrm{mg})$, and from $40-100 \% \mathrm{CH}_{3} \mathrm{CN}$ over $30 \mathrm{~min}$ to get $\mathbf{3}(8.68 \mathrm{mg})$ and $\mathbf{4}(6.50 \mathrm{mg})$.

Compound 1: Red powder; UV $(\mathrm{MeOH}) \lambda_{\max }(\log \varepsilon): 225$ (4.2), 277 (4.2), 466 (3.7) nm; ${ }^{1} \mathrm{H}-\mathrm{NMR}$ (500 MHz, CD $\mathrm{CD}_{3} \mathrm{OD}$ and ${ }^{13} \mathrm{C}-\mathrm{NMR}\left(125 \mathrm{MHz}, \mathrm{CD}_{3} \mathrm{OD}\right)$ : see Tables 1 and 2; HRESI-MS (positive mode): $m / z$ 321.0737, $[\mathrm{M}+\mathrm{Na}]^{+}$(Calcd for $\mathrm{C}_{17} \mathrm{H}_{14} \mathrm{O}_{5} \mathrm{Na}, 321.0739$ ).

Compound 2: Red powder; UV (MeOH) $\lambda_{\max }(\log \varepsilon): 226$ (4.3), 277 (4.3), 465 (3.8) nm; ${ }^{1} \mathrm{H}-\mathrm{NMR}$ (500 MHz, CD $\mathrm{CD}_{3}$ ) and ${ }^{13} \mathrm{C}-\mathrm{NMR}\left(125 \mathrm{MHz}, \mathrm{CD}_{3} \mathrm{OD}\right)$ : see Tables 1 and 2; HRESI-MS (positive mode): $\mathrm{m} / \mathrm{z}$ 335.0894, $[\mathrm{M}+\mathrm{Na}]^{+}$(Calcd for $\left.\mathrm{C}_{18} \mathrm{H}_{16} \mathrm{O}_{5} \mathrm{Na}, 355.0895\right)$.

Compound 3: Red powder; UV (MeOH) $\lambda_{\max }(\log \varepsilon): 228$ (4.4), 275 (4.3), 465 (3.9) nm; ${ }^{1} \mathrm{H}-\mathrm{NMR}$ (500 MHz, CD $\mathrm{CD}_{3}$ ) and ${ }^{13} \mathrm{C}-\mathrm{NMR}\left(125 \mathrm{MHz}, \mathrm{CD}_{3} \mathrm{OD}\right)$ : see Tables 1 and 2; HRESI-MS (positive mode): $\mathrm{m} / \mathrm{z}$ 349.1051, $[\mathrm{M}+\mathrm{Na}]^{+}$(Calcd for $\mathrm{C}_{19} \mathrm{H}_{18} \mathrm{O}_{5} \mathrm{Na}$, 349.1052).

Compound 4: Red powder; UV (MeOH) $\lambda_{\max }(\log \varepsilon): 225$ (4.3), 277 (4.3), 465 (3.8) nm; ${ }^{1} \mathrm{H}-\mathrm{NMR}$ (500 MHz, CD $\left.\mathrm{CD}_{3} \mathrm{O}\right)$ and ${ }^{13} \mathrm{C}-\mathrm{NMR}\left(125 \mathrm{MHz}, \mathrm{CD}_{3} \mathrm{OD}\right)$ : see Tables 1 and 2; HRESI-MS: $m / z$ 349.1051, $[\mathrm{M}+\mathrm{Na}]^{+}$(Calcd for $\mathrm{C}_{19} \mathrm{H}_{18} \mathrm{O}_{5} \mathrm{Na}$, 349.1052).

Cytotoxicity Assays The cytotoxicity of the 1-4 were evaluated in tumor cells lines with the 3-(4,5-dimethylthiazol2-yl)-2,5-diphenyltetrazolium bromide (MTT) assay. ${ }^{7}$ Briefly, the cells were seeded into a 96 -well plate. After $24 \mathrm{~h}$, the medium was replaced with fresh medium ( $2 \%$ fetal calf serum) and a two-fold serial of dilutions of the compounds were added to the wells. After $72 \mathrm{~h}$ of exposure, the medium was removed and MTT was added at a concentration of $5 \mathrm{mg} / \mathrm{mL}$ to each well and the plates were incubated for $4 \mathrm{~h}$ at $37^{\circ} \mathrm{C}$. Dimethylsulfoxide (DMSO, $50 \mu \mathrm{L} /$ well) was added to dissolve the MTT formazan and the optical density of the cells was measured at $570 \mathrm{~nm}$ (OD570) with a microtiter plate reader (Thermo Scientific, MK3). All assays were performed in triplicate on three independent experiments and measurement data were expressed as the mean S.D.

Acknowledgments This work was financially supported by Hubei Agricultural Sciences and Technology Innovation Centre and National Key Technologies R\&D Program (2011BAE06B04). The authors thank Dr. Yucheng Gu (Syngenta Limited, England) for isolation of microorganism and elucidation of compounds. 


\section{References}

1) Tietze L. F., Gericke K. M., Schuberth I., Eur. J. Org. Chem., 2007, 4563-4577 (2007).

2) Hori Y., Abe Y., Nishimura M., Goto T., Okuhara M., Kohsaka M., J. Antibiot., 46, 1069-1075 (1993).

3) Wright A. D., Nielson J. L., Tapiolas D. M., Motti C. A., Ovenden S. P. B., Kearns P. S., Liptrot C. H., Mar. Drugs, 7, 565-575 (2009).

4) Igarashi Y., Trujillo M. E., Martinez-Molina E., Yanase S., Miya- naga S., Obata T., Sakurai H., Saiki I., Fujita T., Furumai T., Bioorg. Med. Chem. Lett., 17, 3702-3705 (2007).

5) Igarashi Y., Yanase S., Sugimoto K., Enomoto M., Miyanaga S., Trujillo M. E., Saiki I., Kuwahara S., J. Nat. Prod., 74, 862-865 (2011).

6) Hu Y. C., Martinez E. D., MacMillan J. B., J. Nat. Prod., 75, 17591764 (2012).

7) Mosmann T., J. Immunol. Methods, 65, 55-63 (1983). 